\title{
Irregular object simplify method based on multi-granularity
}

\author{
Xiaoping Liao ${ }^{1, a}$, Haihua Xiao ${ }^{2, b^{*}}$ and Junyan $\mathrm{Ma}^{3, \mathrm{c}}$ \\ ${ }^{1}$ School of Mechanical engineering Guangxi University Nanning, China \\ ${ }^{2}$ School of Mechanical engineering Guangxi University Nanning, China \\ ${ }^{3}$ School of Mechanical engineering Guangxi University Nanning, China \\ a286269741@qq.com, b754561520@qq.com, ${ }^{\text {c}} 191159191 @ q q . c o m$
}

\begin{abstract}
Keywords: layout design; irregular objects; multi-granularity; simplify method; compactness.
Abstract. In order to improve the local space utilization, this paper researches on simplify method for irregular objects. Simplify method based on multi-granularity is proposed. According to the collision possibility of each object in the initial layout, different granularities are set for different objects. For one object, its global threshold and local threshold can be calculated by granularity. The object mode is determined by the relationship between Vchobj-Vobj and global threshold and the relationship between Vsubmax and local threshold. The object modes include prototype, convex hull and simplified analogue that own some special geometry features. Approaches for special Boolean blocks are set. A system was developed to verity the feasibility of the proposed method. Compared to single granularity simplify method, simplify method based on multi-granularity can improve the layout compactness effectively.
\end{abstract}

\section{Introduction}

Generally, because of the complex geometry of actual objects in 3D engineering layout design, using traditional CAD models of components leads to time-consuming interference checking and volume measurement. Thus some modeling approaches are employed to approximate the geometry of components to shorten the interference checking time. Methods are grouped into two categories, decomposition algorithm and bounding box algorithm.

The basic idea of decomposition algorithm is that the object is gradually broken down into hierarchical structures. Interference checking between complex geometries can be solved by interference checking between simple geometries. Decomposition algorithm includes octree method and finite sphere method (FSM) in 3D or finite circle method (FCM) in 2D. Cagan et al.[1] presented a multi-resolution modeling based on octree decomposition to solve constraints satisfaction and interference checking problem of arbitrary components successfully. For the definition of non-overlapping constraints, Zhang et al.[2] proposed a revised finite circle method that adapts shape changes of elliptical components. Quinlan[3] constructed a hierarchical bounding representation based on spheres for computing the distance between non-convex objects. The distance between objects is determined by computing the distance between pairs of convex components. Gao et al.[4] proposed a new strategy for the efficient integrated layout design of multi-component system. FCM method was used to approximately describe the outline of component. Thus geometry interference problem was solved.

The basic idea of bounding box algorithm is that using simple geometry with large size instead of complex geometric objects for interference checking. The most common bounding boxes include Axis-Aligned Bounding Boxes (AABB), Oriented Bounding Box (OBB), Sphere, Fixed Directions Hulls (FDH) ${ }^{[5]}$. Wang Y et al. ${ }^{[6]}$ adopts AABB algorithm and two-dimensional projection for crudity detection. FDH14 $(\mathrm{k}=14)$ is chosen for further detection. Application in NC machining simulation presented that the algorithm is effective and efficiency. Shi et al. ${ }^{[7]}$ proposed a rapid collision detection algorithm based on the improved theory of Oriented Bounding Box to solve the problem of the low precision of collision detection in the turn-mill combined machining simulation. Tang et al. ${ }^{[8]}$ proposed a new method for collision detecting based on adaptive enclosing ellipsoids. Experiments 
demonstrate that the proposed method can improve the detection efficiency and guarantee the detection precision.

Decomposition algorithm and bounding box algorithm are methods based on single granularity. They can simplify the arbitrary components and improve the detection efficiency. However, most actual objects in engineering layout design own special geometry features in order to realize different functions. Decomposition algorithms and bounding box algorithms ignore the function feature structure of components so that they lead to inefficient in layout design and low utilization. Thus, this paper proposed a simplify method based on multi-granularity.

\section{Object Simplify Method Based on Multi-granularity}

Multi-granularity Set Method. Rubber ball analogy layout method is a typical visual layout design method $^{[9]}$. It is a set of physical movements on the base of many time steps. When the initial layout is given, the probability of collision between two objects in the first time step can be judged by the elastic force. Hence multi-granularity set method can be obtained.

Collision between two objects is detected at the end of the first time step. If there is collision between object $\mathrm{i}$ and object $\mathrm{j}, \mathrm{a}_{\mathrm{ij}}$ equals to 1 , otherwise equals to 0 . Matrix $\mathrm{A}_{\mathrm{ij}}$ is constructed as in (1).

$$
A_{i j}=\left[\begin{array}{lllccc}
0 & a_{12} & a_{13} & \Lambda & a_{1 j} & \Lambda \\
0 & 0 & a_{23} & \Lambda & a_{2 j} & \Lambda \\
\mathrm{M} & \mathrm{M} & \mathrm{M} & \mathrm{M} & \mathrm{M} & \mathrm{M} \\
0 & 0 & 0 & \Lambda & a_{i j} & \Lambda \\
0 & 0 & 0 & 0 & 0 & \Lambda \\
\mathrm{M} & \mathrm{M} & \mathrm{M} & \mathrm{M} & \Lambda & \mathrm{M}
\end{array}\right]
$$

The possibility of collision of each object is

$$
a_{i}=\sum_{j=1}^{n} a_{i j}
$$

Array ai by value in descending order to get a sequence $\left\{a_{1}^{\prime}, a_{2}^{\prime}, \Lambda, a_{i}^{\prime}, \Lambda, a_{n}^{\prime}\right\}$, where

$$
a_{1}^{\prime}=\max \left\{a_{i}\right\}, a_{n}^{\prime}=\min \left\{a_{i}\right\}
$$

Object corresponding to ${ }^{a_{1}}$ get the minimum granularity which equals to 0.The sum of granularities of other objects equals to 1 . The larger the value of ${ }^{a_{i}}$, the smaller of the value of granularity.

Mathematical model of object simplify method based on multi-granularity is

$$
C=\eta_{1} k_{1}+\eta_{2} k_{2}+\cdots+\eta_{n} k_{n}, \sum_{i=1}^{n} \eta_{i} \approx 1, \eta_{i} \geq 0
$$

C is a layout simplify program, $\eta_{i}$ is the simplify granularity of the $i_{\text {th }}$ object. $k_{\mathrm{i}}$ is the $i_{\text {th }}$ object.

Simplify method for one object. The basic idea of the simplify method for one object is as follows. Firstly, calculate the global threshold $\varphi_{\text {global }}$ and local threshold $\varphi_{\text {local }}$ based upon the relationship 
between threshold and granularity $\eta_{o b j}$. Secondly, calculate the values of $\mathrm{V}_{\text {obj }}, \mathrm{V}_{\text {chobj }}$ and $\mathrm{V}_{\text {submax }} \cdot \mathrm{V}_{\text {obj }}$ refers to the volume of object. $\mathrm{V}_{\text {chobj }}$ is the volume of its convex hull. $\mathrm{V}_{\text {submax }}$ refers to the volume of the biggest Boolean block.

generated by Boolean difference operation of convex hull and object. Thirdly, compare the value of $\mathrm{V}_{\text {obj }}-\mathrm{V}_{\text {chobj }}$ and $\varphi_{\text {global }}$ and the value of $\mathrm{V}_{\text {submax }}$ and $\varphi_{\text {local }}$.Compare results decide the object mode that participates layout process. The object mode include prototype, convex hull and simplified analogue that contains some holes or concave features.

Relationship between $\varphi_{\text {global }}$ and granularity $\eta_{o b j}$ and relationship between $\varphi_{\text {local }}$ and $\eta_{o b j}$ are

$$
\varphi_{\text {global }}=\eta_{o b j} V_{o b j}, \quad \varphi_{\text {local }}=\eta_{o b j} V_{s u b_{\max }}
$$

Flowchart of simplify method is illustrated in Fig.1.

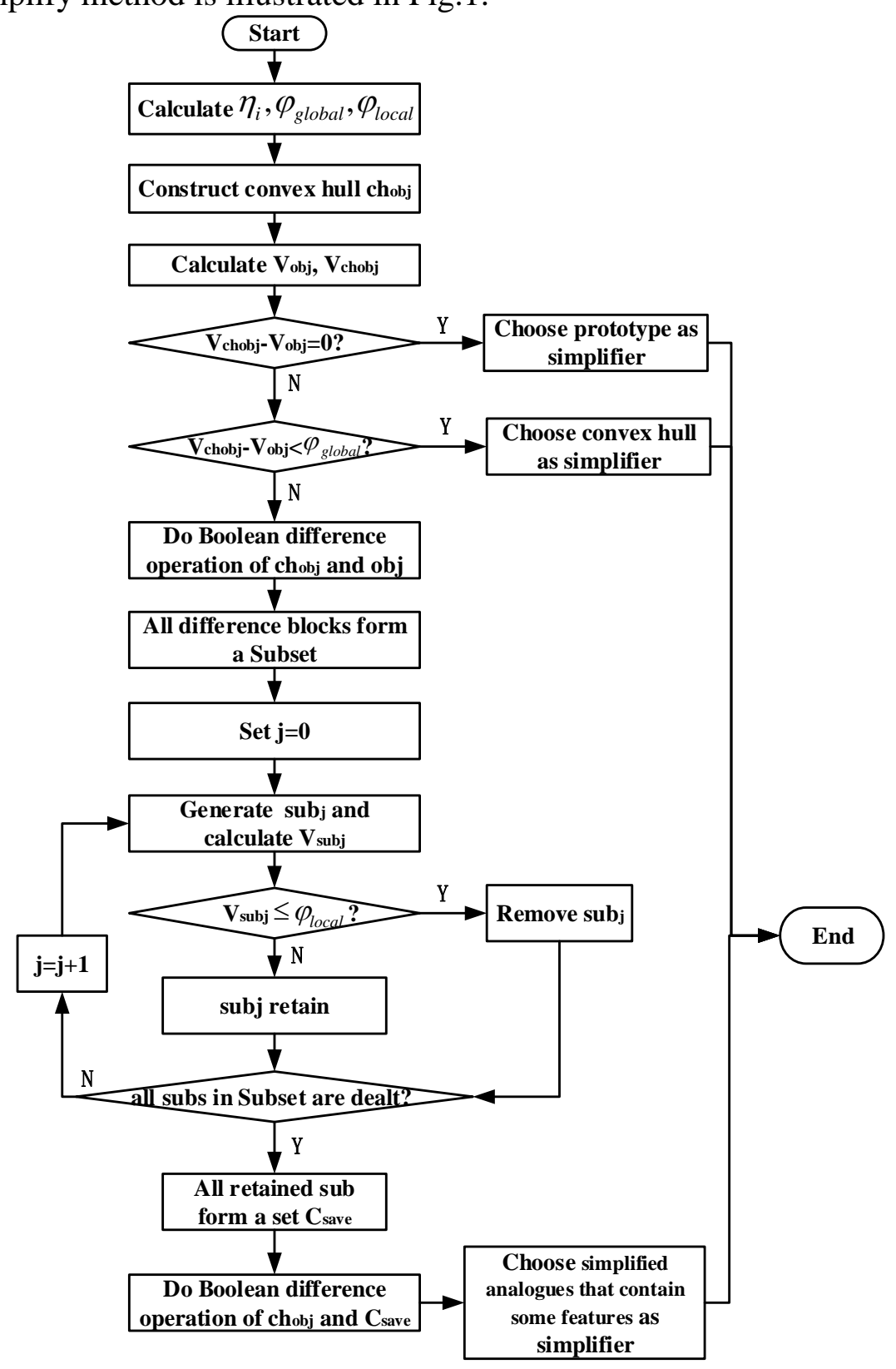

Figure 1 Flowchart of simplify method Algorithm of simplifying the object is as follows.

(1) Calculate the object granularity, global threshold and local threshold.

(2)Construct the object's convex hull as $\mathrm{ch}_{\mathrm{obj}}$, calculate $\mathrm{V}_{\text {chobj }}$ and $\mathrm{V}_{\mathrm{obj}}$. 
(3)Decide the object mode based on the relationship between $\mathrm{V}_{\text {chobj }}$ and $\mathrm{V}_{\text {obj. }}$.

(a)If $\mathrm{V}_{\text {obj }}-\mathrm{V}_{\text {chobj }}=0$, the object is a convex object. Prototype is chosen to join the layout process and the simplify process ends.

(b)If $\mathrm{V}_{\text {obj }}-\mathrm{V}_{\text {chobj }}<\varphi_{\text {global }}$, the object contains small holes or concave features. Convex hull is chosen to be the simplifier and the simplify process ends.

(c)If $V_{c h_{o b j}}-V_{o b j}>\varphi_{g l o b a l}$, the object contains big holes or concave features. Further research is needed, go to step (4).

(4)Do Boolean difference operation of object and its convex hull. Generate Boolean block $s u b_{j}$ which corresponds to the $\mathrm{j}_{\mathrm{th}}$ hole or the $\mathrm{j}_{\mathrm{th}}$ concave feature.

(5) Calculate the value of $V_{s u b_{j}}$, the volume of $s u b_{j}$, and determine whether $s u b_{j}$ should retain or not according to the relationship between $V_{\text {sub }}$ and $\varphi_{\text {local }}$.

(a)If $V_{\text {sub }}<\varphi_{\text {local }}, s u b_{j}$ is small, that's it, the $\mathrm{j}_{\text {th }}$ hole or the $\mathrm{j}_{\mathrm{th}}$ concave feature is small. The Boolean block should be removed.

(b)If $V_{\text {sub }}>\varphi_{\text {local }}, \quad s u b_{j}$ is large, that means, the $\mathrm{j}_{\mathrm{th}}$ hole or the $\mathrm{j}_{\text {th }}$ concave feature is large. The Boolean block should be retained.

(6) Repeat step (5) until all the Boolean blocks are dealt. All the reserved Boolean blocks form a set $C_{\text {save }}$. Do Boolean difference operation of $c h_{o b j}$ and $C_{\text {save }}$. The result is the simplified analogue that the system needs.

Special Boolean block treatment. The difference between volumes is used as the basis of object simplification. Principle of this method is simple and the result is relatively accurate. But this method must make the appropriate special treatment for some special Boolean blocks, such as slender blocks and annular blocks.

A slender block is a block with a small sectional area and long length, shown as Fig.2.

Figure 2 A slender block

When ratio between the inner radius and wall thickness is larger than a given value, the object is an annular block, as shown in Fig.3.

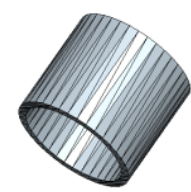

Figure 3 An annular block

Holes or concave features that correspond to slender blocks or annular blocks should retain according to simplify method mentioned in section 2.2. Other objects have little probability to enter these holes or concave areas. These holes or concave areas make the layout process more complicated. Holes or concave features that correspond to slender blocks or annular blocks should remove in the actual treatment.

\section{Examples and Analysis}

Examples. Objects can be classified into three classes. Class A is regular objects. No matter what the granularity is, prototype is used to participate the layout process, such as a cylinder, as shown in Fig.4. 


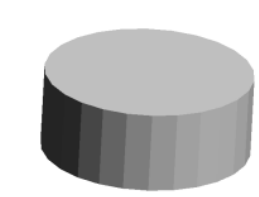

Figure 4 Class A

Class B has a hole or only one concave features, as shown in Fig.5, (a) is the prototype of object B, (b) is its convex hull. Volume of B is $18.0336 \mathrm{~mm}^{3}$, volume of its convex hull is $21.9908 \mathrm{~mm}^{3}$. When $\eta \leq 0.22$ is true, (a) is used for layout, otherwise (b) is used for layout.

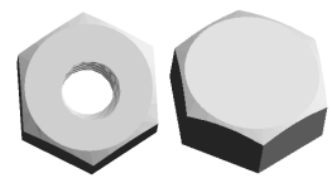

(a) (b)

Figure 5 Class B and its convex hull

Class C has many holes or concave features, as shown in figure 6 . Volume of $\mathrm{C}$ is $1958.63 \mathrm{~mm}^{3}$ and volume of its convex hull is $3000.00 \mathrm{~mm}^{3}$. When $\eta>0.53$, simplifier (c) is used for layout. When $\eta \leq 0.53$, relationship between volume of Boolean blocks and local threshold should be considered. Volume of concave feature number 1 is $900.000 \mathrm{~mm}^{3}$. Volume of hole number 1 is $139.456 \mathrm{~mm}^{3}$. When $\eta \leq 0.15$, (a) is used for layout. When $0.15<\eta \leq 0.53$, (b) is used for layout.

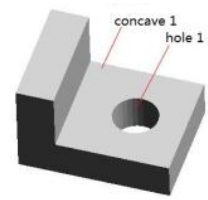

(a)

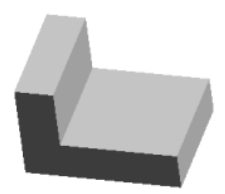

(b)

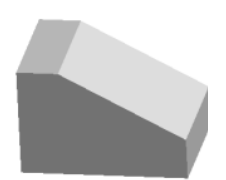

(c)

Figure 6 Class $\mathrm{C}$ and its simplifiers

Initial layout is as shown in Fig.7. Time step is set as $1 / 60 \mathrm{~ms}$. At the end of the first time step, the layout process is as shown in Fig.8.

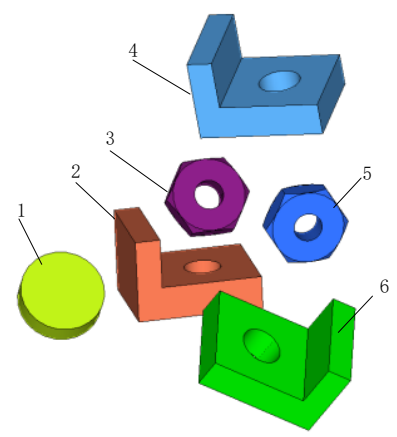

Figure 7 Initial layout with prototype

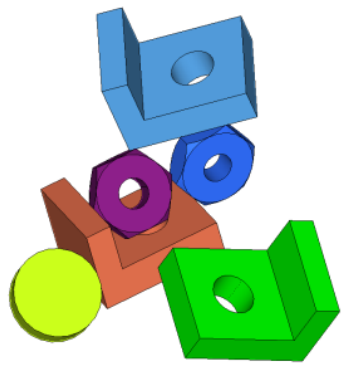

Figure 8 Layout of the first time step

$a_{i}$ and $\eta_{i}$ can be calculated. The results are shown in Table 1 . 


\begin{tabular}{|c|r|r|r|}
\hline $\begin{array}{c}\text { Object } \\
\text { ID }\end{array}$ & $a_{i}$ & $a_{i}^{\prime}$ & $\eta_{i}$ \\
\hline 1 & 1 & 4 & 0.2352 \\
\hline 2 & 4 & 1 & 0.0588 \\
\hline 3 & 3 & 2 & 0.1176 \\
\hline 4 & 2 & 3 & 0.1764 \\
\hline 5 & 2 & 3 & 0.1764 \\
\hline 6 & 1 & 4 & 0.2352 \\
\hline
\end{tabular}

Table 1 Object and their $a_{i}, \eta_{i}$

Different objects has different granularities, initial layout with simplified objects is shown in Fig.9.

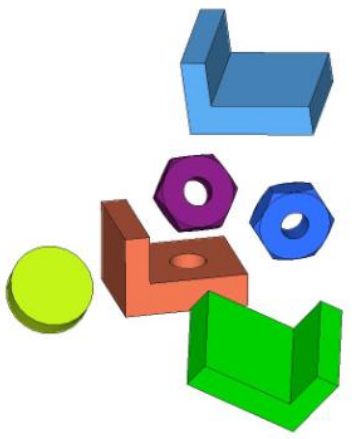

Figure 9 Initial layout with simplified objects

Analysis. Compactness in 3D layout design is measured as the ratio between the sum of the volumes of all objects and the volume of the layout space ${ }^{[10]}$.

Simplify objects by single granularity method (such as convex hull) and the proposed multi-granularity method are used for layout design separately. Compactness under different methods are compared in Fig. 10.

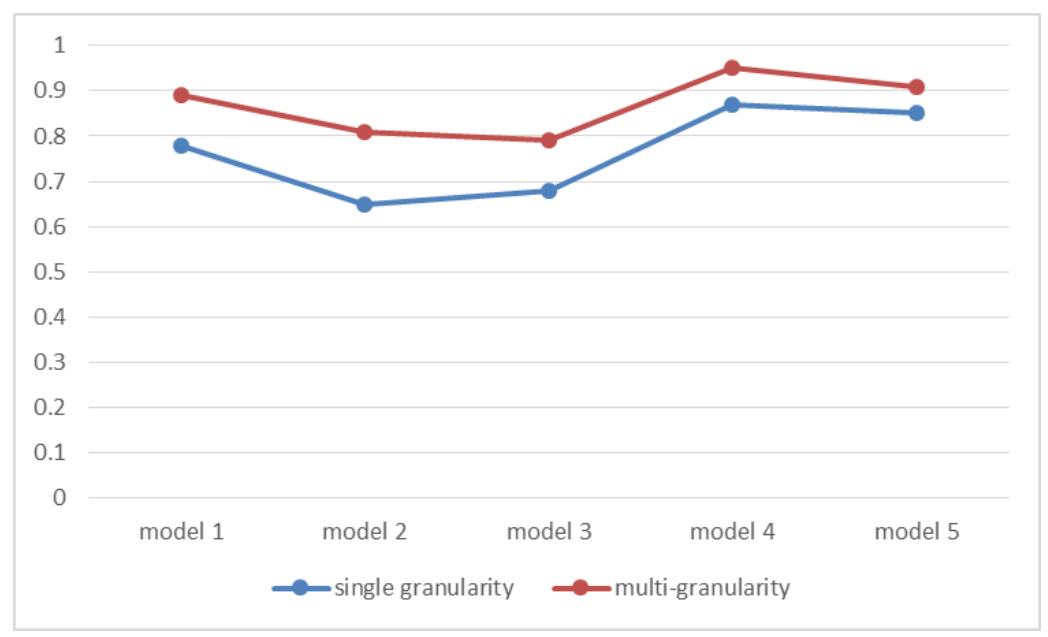

Figure 10 Comparison picture of compactness

From Fig.10, we can see that multi-granularity method get the higher compactness than single granularity. 


\section{Summary}

Irregular object simplify method based on multi-granularity is proposed. Granularity set method, simplify method for one object and special Boolean block treatment method are described in detail. Simplified objects based on single granularity and multi-granularity are used for layout design separately. Comparison result verify the feasibility and effectiveness of the proposed method. It provides the foundation for digital design of irregular objects layout. The outline recognition of objects is to be further researched so that the compactness of layout can be further improved.

\section{ACKNOWLEDGMENT}

We gratefully acknowledge the support of National Natural Science Foundation of China (NO.51265002).

\section{References}

[1] Cagan J, Degentesh D, Yin S. A simulated annealing-based algorithm using hierarchical models for general three-dimensional component layout[J]. Computer-aided design, 1998, 30(10): 781-790.

[2] Zhang J, Zhang W H, Zhu J H, et al. Integrated layout design of multi-component systems using XFEM and analytical sensitivity analysis[J]. Computer Methods in Applied Mechanics and Engineering, 2012, 245: 75-89.

[3]Quinlan S. Efficient distance computation between non-convex objects[C]//Robotics and Automation, 1994. Proceedings., 1994 IEEE International Conference on. IEEE, 1994: 3324-3329.

[4] Gao H H, Zhu J H, Zhang W H, Zhou Y.An improved adaptive constraint aggregation for integrated layout and topology optimization[J]. Computer Methods in Applied Mechanics and Engineering,2015, 289:387-408.

[5] Ericson C. Real-time collision detection[M]. CRC Press, 2004.

[6] Wang Y, Hu Y, Fan J, et al. Collision Detection Based on Bounding Box for NC Machining Simulation[J]. Physics Procedia, 2012, 24: 247-252.

[7] Shi X H, Qiao L H, Zhu Z W. Algorithm of collision detection based on improved oriented bounding box[J]. Journal of Hunan University(Natural sciences), 2014, 41: 26-31.

[8] Tang Y, Yang S S, Lu M Y, et al. Collision detection for cloth based on adaptive enclosing ellipsoids[J]. Journal of computer-aided design \& computer graphics, 2013, 25: 1589-1596.

[9] Ma J Y,Long F Y,Liao X P,et al.Digital modeling and movement resolution of rubber balloon analogy for 3D packing problem[J].Advanced Materials Research, 2013, 753: 1670- 1674.

[10] Wei J X, Liao X P, Tian B B, et al. The Research on Construction of Convex Hull Plus Rubber Ball Analog Layout Model [J]. Machinery Design \& Manufacture, 2015 (5):220-223. 